\title{
Estimation of Sediment Trap Efficiency in Reservoirs -An Experimental Study -
}

\author{
N.M.T.K. Revel, L.P.G.R. Ranasiri, R.M.C.R.K. Rathnayake and K.P.P. Pathirana
}

\begin{abstract}
Reservoir sedimentation has become one of the major problems facing water resources development projects in many countries around the world. However, only a limited number of studies have been reported in this field, particularly addressing the trap efficiency of reservoirs. In addition, even the available studies in this area have considered only few parameters governing reservoir sedimentation. As a result, the available knowledge on trap efficiency is not very well defined. The Brune curve [4] is being widely used for estimating trap efficiency of reservoirs at present, but it has several limitations, as it considers only the reservoir capacity and inflow ratio for estimating the trap efficiency. The objective of this study is to formulate an improved methodology for estimating reservoir sedimentation through laboratory experiments. A small-scale laboratory model was set-up to represent a reservoir and a series of tests were conducted by varying the inflow rate, inflow sediment concentration, reservoir capacity and the outflow rate. The experimental results were compared with values obtained from available theories and it was found that they are not very much in agreement with many of the existing theories which are mostly based on a limited number of parameters. A comprehensive data analysis was performed using dimensional analysis to develop an improved relationship to estimate reservoir sedimentation incorporating many parameters governing the problem. However, the applicability of the proposed method is still limited only to reservoirs with continuous spilling conditions. In addition, only one type of sediment gradation $\left(\mathrm{d}_{50}\right)$ was used in the experimental runs and thus, the effect of sediment sizes is not well represented in this method. However, the relationship developed in this study could be further improved by conducting more experimental runs by varying few other parameters which have not been considered in the present study.
\end{abstract}

Keywords: $\quad$ Reservoir sedimentation, Trap efficiency, Release efficiency, Sediment inflow rate

\section{Introduction}

Reservoir sedimentation is a severe problem in the management of water resources development projects in many countries around the world, as it reduces the original capacity of the reservoirs significantly affecting the irrigation, hydropower and drinking water supply, flood control and recreational activities. The Rantambe reservoir in Sri Lanka is one of the reservoirs which is severely affected by sedimentation. Due to lack of reservoir management practices such as periodical sediment flushing, reservoir sediment routing and improvement of catchment protection to reduce soil erosion, the sedimentation of reservoirs is now becoming a major threat to many countries around the world. Though it is also becoming a major threat to engineers who are responsible for managing water resources projects at present, a limited number of studies has been reported in the field of reservoir sedimentation. Brune [4] and Churchill [5] have proposed empirical methods to evaluate trap efficiency of reservoirs. However, the applicability of these methods is highly limited as their formulations consider only few parameters which affect reservoir sedimentation. This paper presents an experimental study carried out in formulating an improved methodology to estimate reservoir sedimentation.

\section{Literature Review}

Very few studies have been reported in literature on reservoir sedimentation and most of them are based on analytical methods. In general, the amount of sediment accumulated in a reservoir is expressed as a percentage of the inflow sediment quantity which is called sediment Trap Efficiency(TE).

Eng. N.M.T.K. Revel, B.Sc. Eng. (Hons), Department of Civil Engineering, University of Peradeniya.

Eng. L.P.G.R. Ranasiri, B.Sc. Eng. (Hons), Department of Civil Engineering, University of Peradeniya.

Eng. R.M.C.R.K. Rathnayake, B.Sc. Eng. (Hons),

Department of Civil Engineering, University of Peradeniya.

Eng.(Prof.) K.P.P. Pathirana, B.Sc. Eng. (Hons.)

(Peradeniya), M.Eng., Ph.D., C.Eng., FIE(Sri Lanka), MICE

(London), Professor of Civil Engineering, Department of Civil Engineering, University of Peradeniya. 


$$
T E=\frac{\text { Amount of sediment deposits }}{\text { Amount of sediment in inflow }} \ldots . .
$$

Brune[4] has developed a curve relating TE to capacity $(V)$ to annual average inflow $(I)$ ratio as presented in Figure 1, extracted from Reservoir Sedimentation Handbook by Morris et.al[7]. Brune's curve has been widely used to estimate the sedimentation in reservoirs.

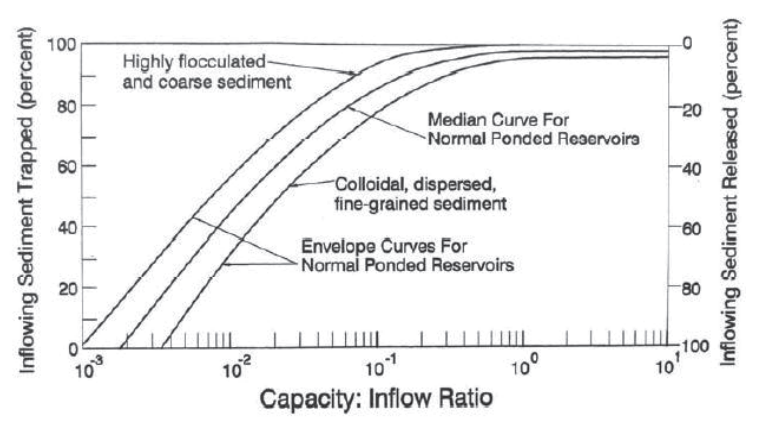

Figure 1 -Brune curve [4]

This curve can also be presented as follows:

$$
T E=100 \times 0.97^{0.19^{\log \left(\frac{V}{I}\right)}} .
$$

where, $T E=$ trap efficiency; $V=$ reservoir capacity; and $I=$ annual average inflow.

Brune [4] had considered only two parameters in his formulation namely; the reservoir capacity and the average annual inflow rate and many other parameters that affect the reservoir sedimentation have not been represented in his method. In addition, Brune [4] had used only normally ponded reservoirs in deriving this empirical relationship. However, his method is found to be not accurate enough in estimating sedimentation quantities in reservoirs with highly variable inflows, as the sediment trapped in the reservoir will be very much influenced by the sediment inflow rate.

Further, Siyam [9] has shown that Brune's [4] curve is a special case of a more general trap efficiency function given by the following equation;

$$
T E=100 e^{\beta\left(\frac{V}{I}\right)} .
$$

where, $\beta$ is a sedimentation parameter that reflects the reduction in the reservoir storage capacity due to sedimentation.

Siyam [9] has also demonstrated that the above equation with values of $\beta=0.0055,0.0079$ and 0.015 would well-define the upper, median and lower Brune's [4] curves respectively as shown in Figure 1.

Churchill [5]developed a graphical relationship between Release Efficiency (RE) and Sedimentation Index $(S I)$ as shown in Figure 2 which is extracted from Reservoir Sedimentation Handbook byMorris et.al[7].

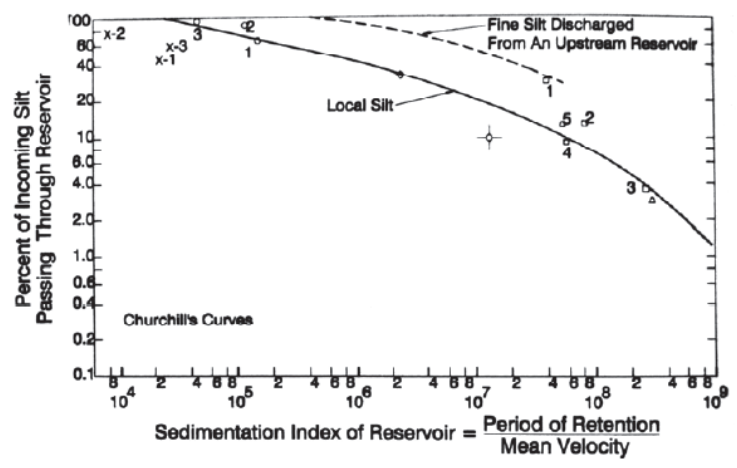

Figure 2 - Churchill curve [5]

Sedimentation index can be defined as follows:

$$
S . I=\frac{\text { Detention time }}{\text { Mean velocity }}
$$

$$
S . I=\frac{(\text { Reservoir capacity })^{2} \times g}{(\text { Reservoir inflow })^{2} \times \text { Reservoir length }} .
$$

Churchill has also considered only a limited number of parameters such as the detention time and mean velocity, omitting other parameters affecting the reservoir sedimentation. But Trimble et.al [11] concluded that the Churchill[5] method appears to produce a more realistic estimate of the sediment yields than from what is estimated the Brune's method [4], for reservoirs receiving sediments from an upstream reservoir. Borland[2] has also concluded that the Churchill[5] curve is somewhat superior to Brune's curve[4],in estimating reservoir sedimentation.

Verstraetenet.al [6] have stated that although the use of the Churchill curves [5] may give a better prediction of TE compared to what is obtained from the Brune curves [4], it is very difficult to obtain the necessary input data for calculating the sedimentation index for Churchill[5] curve. This is probably the reason why Brune's [4] approach is used so extensively as opposed to that of Churchill [5].

Brown [3] also has also presented a correlation for TE incorporating the Capacity $(V)$ to Watershed $(W)$ ratio as illustrated in Figure 3. 


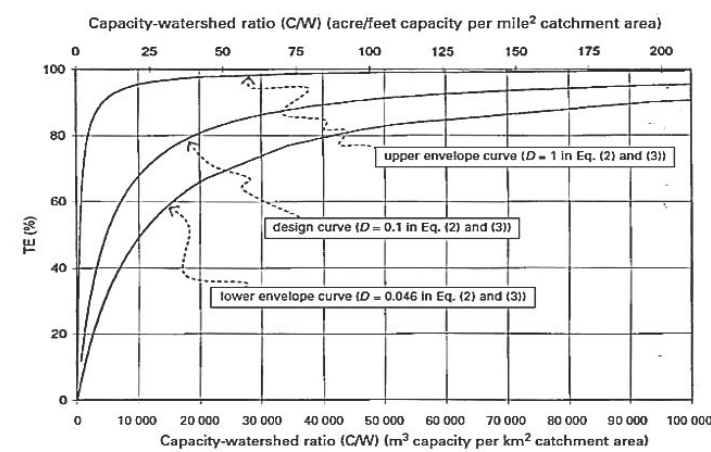

Figure 3 - Brown's curve [3]

The relationship can also be expressed as:

$$
T E=100\left(1-\frac{1}{0.0021 \frac{D \times V}{W}}\right)
$$

where, $V=$ reservoir storage capacity expressed in $\mathrm{m}^{3}$; and $W=$ catchment area expressed in $\mathrm{km}^{2}$. $D$ is a coefficient whose values range from 0.046 to 1 , with a mean value of 0.1 .

Here, Brown [3] has also considered the capacity $(V)$ and watershed area $(W)$ of the reservoir when a more significant number of parameters affect the reservoir sedimentation.

Harbor et al[8] have carried out experimental investigations on reservoir sedimentation and derived a curve which is similar to the Brune curve[4]. This relationship is given in Eq. (7).

$$
T E=-22+\left[\frac{119.6 \frac{V}{I}}{0.012+\left(1.02 \frac{V}{I}\right)}\right] \ldots \ldots \ldots(7)
$$

Toniolo et al [11] have also carried out several experiments on reservoir sedimentation, but their results appear to be very preliminary and have failed to produce any conclusive results.

Bashar et al [1] have carried out a bathymetric survey on Roseires reservoir which is located in Sudan, taking the baseline as the design storage capacity of the reservoir at different levels in 1966 and compared the results so obtained with values obtained using both Brune [4] and Churchill [5] methods. He has concluded that both Brune [4] and Churchill [5] methods overestimate the trap efficiency. Lewis et al [10] have also stated about the overestimation that results from using both Brune [4] and Churchill [5] curves.

\section{Methodology}

\subsection{Experimental Set-up}

The experimental set-up consists of an overhead tank with dimensions of $1.2 \mathrm{~m} \times 1.2$ $\mathrm{m} \times 1 \mathrm{~m}$, which feed sediment laden water into a reservoir, a sediment agitator to keep sediments in suspension mode inside the overhead tank and a separate tank $0.5 \mathrm{~m}$ high, 1 $\mathrm{m}$ wide and $2 \mathrm{~m}$ long, to represent the reservoir. The sediment agitator consists of an electric motor, a vertical shaft with horizontal wings and a stand to fix the motor. Figure 4 shows a schematic diagram of the experimental set-up.

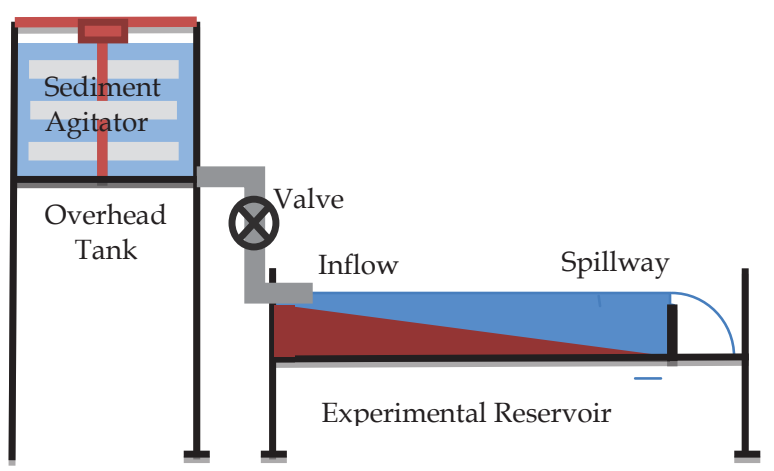

Figure 4 - Schematic Diagram of Experimental Set-up

The rectangular tank was modified by placing four timber plates at the four comers of the tank in order to avoid water stagnation at those corners. The reservoir bed was made linearly sloping towards the dam using compacted soil to represent the model much closer to a real reservoir. The dam was made out of timber which can be moved back and forth to change the reservoir capacity $(V)$. The actual photograph of the experimental set-up is presented in Figure 5.

The following important features were available in the experimental set-up:

- Possibility of controlling the inflow rate of sediment laden water to the reservoir by using a gate value,

- Ability to change the reservoir capacity by adjusting the downstream boundary (dam) of the model reservoir.

- Possibility of changing the spillway length by fixing different sizes of spillway moulds on the dam. In this way, outflow from the reservoir could be changed. 
- Possibility of changing inflow sediment concentration by adding different quantities of sediments to the overhead tank and mixing them with the agitator fixed to this tank.

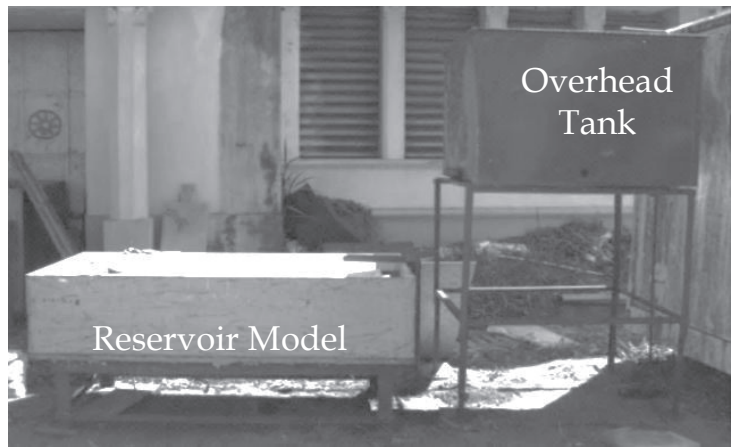

Figure 5 -Experimental Set-up

A reddish sandy soil which passed through a 1 $\mathrm{mm}$ sieve was used as sediments for the proposed tests. Figure 6 illustrates the particle size distribution of the sediments mixed with the inflow to the reservoir. The mean diameter $\left(d_{50}\right)$ of the soil was found to be $0.02 \mathrm{~mm}$.

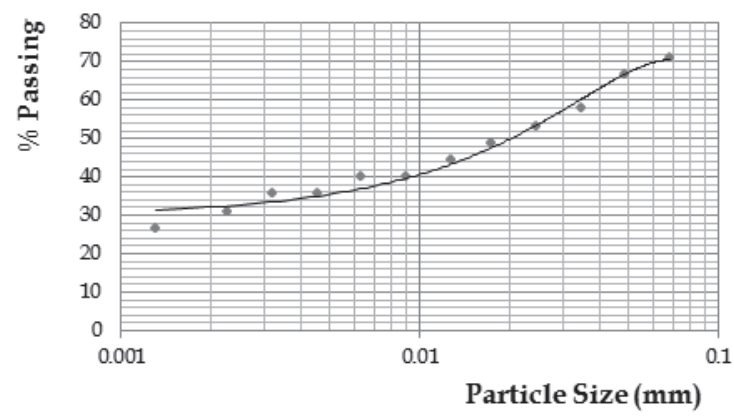

Figure 6 - Particle Size Distribution of the Sediments

\subsection{Experimental Procedure}

The gate valve connected to the outflow pipe of the overhead tank was first adjusted to release water at a specified flow rate to the reservoir and it was allowed to spill over a pre-set length of spillway. The experiment was carried out for a duration of one hour. During the experiments, six sediment samples were taken from the inflow at different times to get the averaged sediment concentration. After the experiments were over, the sediments deposited on the reservoir bed were collected and quantified. This procedure was repeated for three different reservoir capacities $97.1 l$, $119.5 l$ and $144.0 l$, while varying the inflow sediment concentration from $0.10 \mathrm{~kg} / \mathrm{m}^{3}$ to $1.20 \mathrm{~kg} / \mathrm{m}^{3}$. In addition, inflow rates were also changed from $200 \mathrm{ml} / \mathrm{s}$ to $400 \mathrm{ml} / \mathrm{s}$ and two spillway lengths of $200 \mathrm{~mm}$ and $300 \mathrm{~mm}$ were used to control the outflow rate from the reservoir.

\section{Results and Discussion}

\subsection{Experimental Results}

The trapped sediment weight $\left(W_{s}\right)$ was plotted against the modelled parameters as shown in Figure 7, Figure 8, Figure 9 and Figure 10.

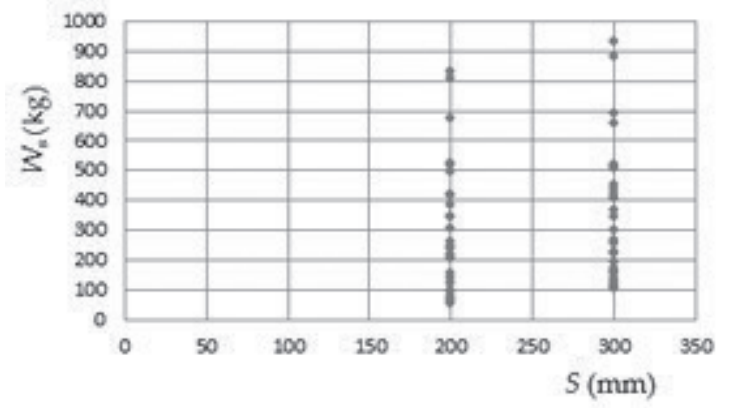

Figure 7 - Variation of $S$ versus $W_{s}$

Experiments were conducted for two different spillway lengths, viz., $200 \mathrm{~mm}$ and $300 \mathrm{~mm}$ and the results are shown in Figure 7 which shows the trapped sediment weight $\left(W_{s}\right)$ increasing slightly as the spillway length $(S)$ increases.

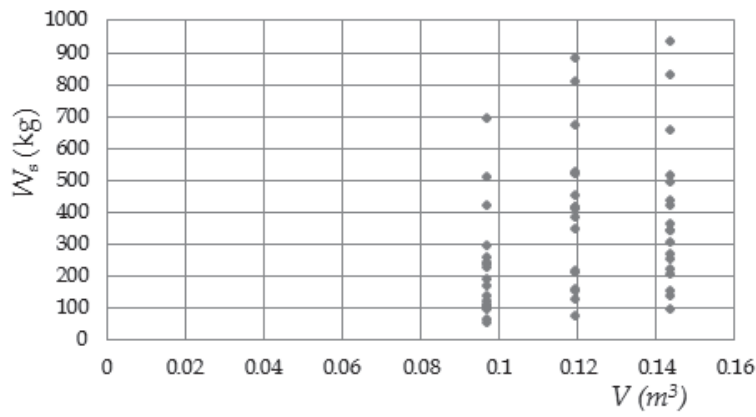

Figure 8 - Variation of $V$ versus $W_{s}$

The trapped sediment weight $\left(W_{s}\right)$ has been plotted against the reservoir capacity $(V)$ in Figure 8 in which the figure indicates the increment of $W_{s}$ with $V$.

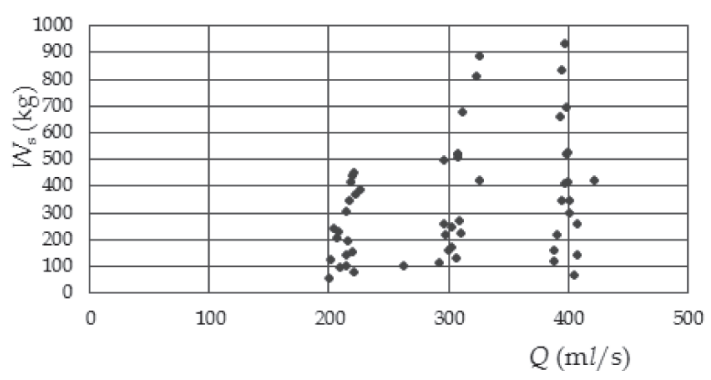

Figure 9 - Variation of $Q$ versus $W_{s}$

A graphical representation of trapped sediment weight $\left(W_{s}\right)$ versus inflow rate $(Q)$ is presented 
in Figure 9. It is not possible to find any correlation here indicating that there are other parameters that affect the sedimentation process.

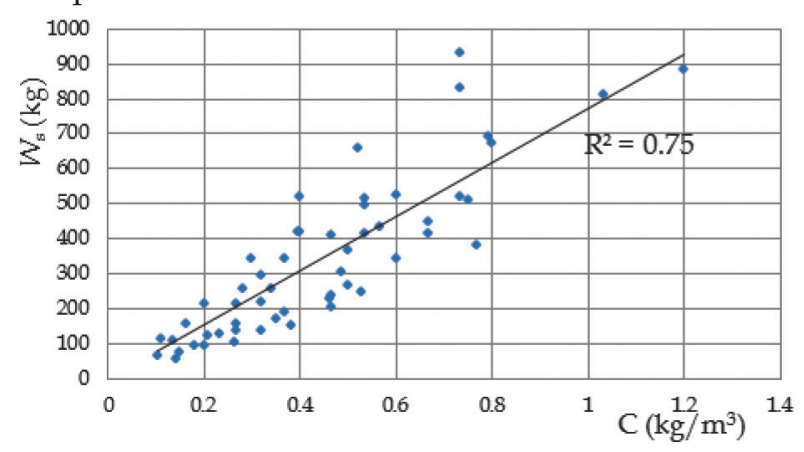

Figure 10 - Variation of $C$ versus $W_{s}$

Figure 10 gives a good correlation between trapped sediment weight $\left(W_{s}\right)$ and the inflow sediment concentration (C). It clearly suggests that the trapped sediment weight $\left(W_{s}\right)$ is influenced to a great deal by the inflow sediment concentration $(C)$.

\subsection{Comparison of Experimental Results with Brune and Churchill Curves}

Figure 11 illustrates the comparison of experimental data with the Brune curve [4]. This graph clearly shows that the experimental data is not very much in agreement with the Brune's curve [4].Trap Efficiencies (TE) estimated from the experimental results are mostly located below the Brune's curve [4], indicating that TE's are somewhat underestimated when compared to Brune curve.

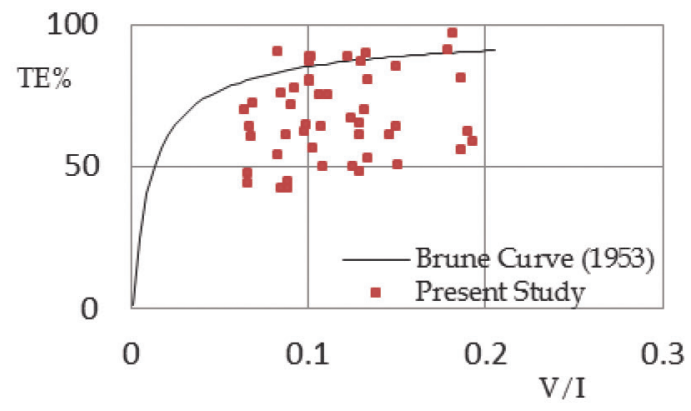

Figure 11 - Comparison of experimental results with the Brune curve [4]

Though the experimental data is very much scattered, it can be seen that there is a trend for $T E$ to increase with the $V / I$ ratio, similar to the Brune curve [4]. It could also be noted that there can be more than one value of TE for a given $V / I$ ratio, indicating that there can be other parameters affecting TE.
Figure 12 shows the comparison of experimental results with the Churchill curve [5].In this case also, the data points are scattered within a narrow region but offers a fare agreement with the curve than with the Brune curve. However it can be observed that more than $50 \%$ of the results below the Churchill curve.

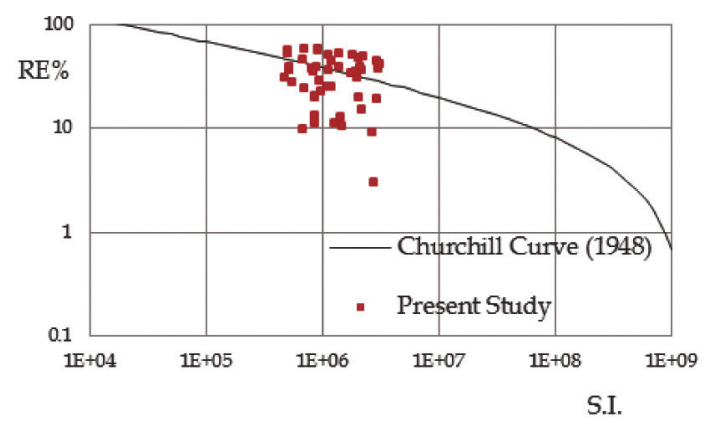

Figure 12 -Comparison of the experimental results with Churchill curve [5]

It is important to highlight here that a few other researchers have also reported about the overestimation of TE by the Brune [4] and Churchill [5] methods based on their studies (Bashar et al [1] and Lewis et al [10]).

As neither Brune's[4] nor Churchill's [5] methods has given a reasonable relationship for the experimental data obtained in this study, a fresh data analysis was adopted using Dimensional Analysis.

\subsection{Dimensional Analysis}

The dry weight of sediment deposited $\left(\mathrm{W}_{\mathrm{s}}\right)$ in a reservoir is affected by the following parameters,

$$
W_{s}=f\left\{\mu, d_{50}, Q, S, L, t, V, g, \varepsilon, \rho, \rho_{s}, C\right\} \ldots
$$

where,

$\mu \quad=$ Dynamic viscosity of water [ML $\left.\mathrm{ML}^{-1} \mathrm{~T}^{-1}\right]$

$d_{50}=$ Mean diameter of sediment particles [L]

$Q \quad=$ Inflow to the reservoir $\left[\mathrm{L}^{3} \mathrm{~T}^{-1}\right]$

$S \quad=$ Weir length (Spillway length) [L]

$C=$ Sediment concentration $\left[\mathrm{ML}^{-3}\right]$

$t \quad=$ Time $[\mathrm{T}]$

$V \quad=$ Reservoir volume [ $\left.\mathrm{L}^{3}\right]$

$g \quad=$ Gravitational acceleration $\left[\mathrm{LT}^{-2}\right]$

$\varepsilon \quad=$ Bed roughness [L]

$L \quad=$ Reservoir length [L]

$\rho=$ Density of water $\left[\mathrm{ML}^{-3}\right]$

$\rho_{S} \quad=$ Density of sediments [ML-3]

The capacity of the model reservoir $(V)$ used for the present study is dependent only on the 
reservoir length $(L)$. Therefore, the parameter $L$ is removed from the above list while retaining the volume term. $\varepsilon$ is removed as the reservoir bed in the experimental setup has been a smooth surface. The term $\rho_{s}$ is also replaced by adding a term $\gamma^{*}$ which is given by $\gamma^{*}=\left(\rho_{s}-\rho\right) g$.

Then,

$$
W_{s}=f\left\{\mu, d_{50}, Q, S, t, V, \gamma^{*}, \rho, \rho_{s}, C\right\} \ldots
$$

Hence, the non-dimensional terms could be formulated as given below:

$$
\frac{\rho g W_{s}}{\mu^{2}}=f\left\{\begin{array}{l}
\frac{\rho^{2 / 3} g^{1 / 3} d_{50}}{\mu^{2 / 3}}, \frac{\rho^{2 / 3} g^{1 / 3} S}{\mu^{2 / 3}}, \frac{\rho^{2} g V}{\mu^{2}} \\
\frac{\rho^{1 / 3} g^{2 / 3} t}{\mu^{1 / 3}}, \frac{C}{\rho}, \frac{\gamma^{*}}{\rho g}, \frac{\rho g}{\mu^{1 / 3} \rho^{5 / 3}}
\end{array}\right\}
$$

As experimental duration ( $t$ ) and sediment density $\left(\rho_{s}\right)$ were not varied during this study, the corresponding dimensionless terms are considered to be constants, and they will not affect the amount of deposited sediments in the reservoir for the present study. Hence, the terms; $\quad \frac{\rho^{2 / 3} g^{1 / 3} d_{50}}{\mu^{2 / 3}}, \frac{\gamma^{*}}{\rho g}$ and $\frac{\rho^{1 / 3} g^{2 / 3} t}{\mu^{1 / 3}}$ are removed from the analysis.

\subsection{Improved correlations among model parameters}

The non-dimensional term representing the trapped sediment weight $\left(W_{s}\right)$ was plotted against the other non-dimensional terms representing other model parameters which were obtained from the dimensional analysis.

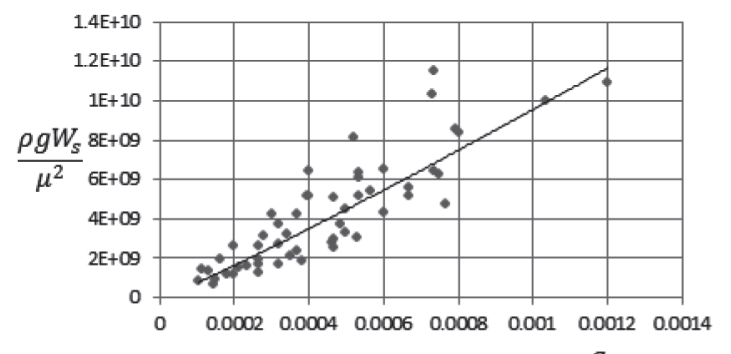

$\frac{C}{\rho}$

Figure 13 - Variation of non-dimensional terms representing $W_{s}$ and $C$

It has already been noted that the inflow sediment concentration has a dominant effect on the trapped sediment amount over the other model parameters. Figure 13 shows the relationship between the non-dimensional term representing inflow sediment concentration $(C)$ and the trapped sediment weight $\left(W_{s}\right)$.

Further combining the terms, inflow sediment concentration $(C)$ and water inflow $(Q)$ gives the sediment inflow rate $\left(I_{s}\right)$ and this term has shown a good correlation with $W_{s}$ showing a tendency to increase as illustrated in Figure14.

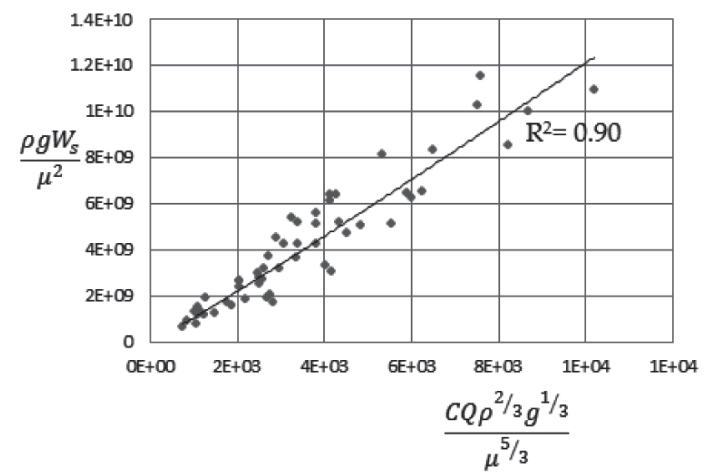

Figure 14 -Variation of non-dimensional terms representing $W_{s}$ and $C Q$

Figure14 shows the effect of inflow sediment rate $\left(I_{s}\right)$ on the trapped sediment weight $\left(W_{s}\right)$ in the reservoir. Combining all of the model parameters, a new relationship was derived as illustrated in Figure 13, where the amount of trapped sediments in the reservoir can be estimated using all the parameters considered in this study. This new formulae incorporates the reservoir capacity $(V)$, sediment inflow rate $\left(I_{s}\right)$, and spillway length $(S)$ which is directly correlated to the outflow from the reservoir. This relationship indicates that the trapped sediment weight $\left(W_{s}\right)$ will reach a maximum value as the term $\left(I_{s} . V / S^{1 / 3}\right)$ increased.

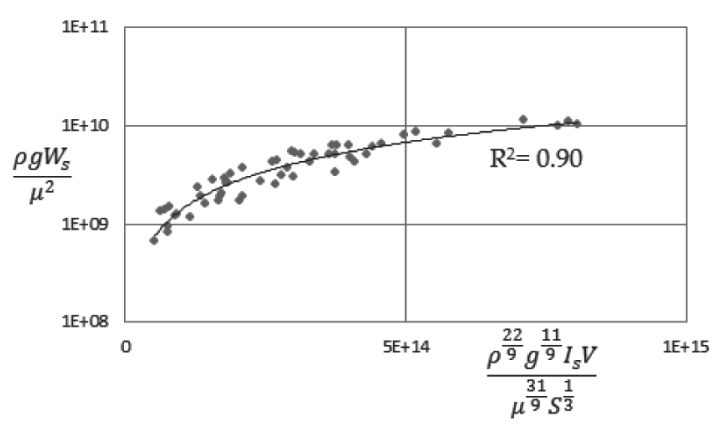

Figure 15 -Variation of trapped sediment weight $\left(W_{s}\right)$ with the model parameters

Figure 15 suggests an equation as shown in Equation 11.

$\frac{\rho g W_{s}}{\mu^{2}}=3 \times 10^{-5}\left(\frac{\rho^{22 / 9} g^{11 / 9}}{\mu^{31 / 9}} \times \frac{I_{s} V}{S^{1 / 3}}\right)^{0.9813}$ 
Equation 11 shows the relationship between the non-dimensional terms representing the trapped sediment weight $\left(W_{s}\right)$ and the combined non-dimensional term of the model parameters. Sediment inflow rate $\left(I_{s}\right)$ and reservoir capacity $(V)$ present a direct proportionality to the trapped sediment weight $\left(W_{s}\right)$, while the spillway length $(S)$ is inversely proportional.

\section{Conclusions and Recommendations}

An experimental investigation was conducted for investigating the sedimentation of reservoirs and particularly, on the trap efficiency of reservoirs. Based on a series of test results obtained, an improved correlation was derived among the parameters governing the reservoir sedimentation. Equation 11 presents a relationship between the non - dimensional terms representing the trapped sediment weight $\left(W_{s}\right)$ and the other model parameters such as sediment inflow rate $\left(I_{s}\right)$, reservoir capacity $(V)$ and spillway length $(S)$.

It can also be found that the inflow sediment rate $\left(I_{s}\right)$ is the most governing parameter for the sedimentation of the reservoir according to the present study.

Equation 11 can be used with a reasonable accuracy to estimate the amount of sediments deposited in a reservoir using other parameters. However, the applicability of the proposed method is still limited only to the reservoirs with a continuous spilling condition. In addition, only one type of sediment gradation $\left(d_{50}\right)$ was used in the experimental runs and thus, the effect of sediment sizes is not well represented in this method. However, the relationship developed during this study could be further improved by conducting more experimental runs by varying few other parameters which have not been considered in the present study.

As the present study concentrates only on the continuous spilling condition of reservoirs, several experiments need also to be conducted with non-spilling conditions. Further studies would also be needed to account for the operating rules of reservoirs where sediment outflow from the reservoir will be partly restricted. The effect of the reservoir shape and its orientation which are also important factors affecting the amount of sediment trapped in the reservoir, needs to be investigated.

\section{References}

1. Bashar, K. E., and Eltayeb Ahmed, 2010. Sediment Accumulation in Roseires Reservoir. Nile Basin Water Science \& Engineering Journal, Vol.3, (Issue 3), pp. 46-55.

2. Borland, W. M., 1971: Reservoir Sedimentation. In Shen, H.W., editor, River mechanics. Vol. II, Fort Collins, CO: Colorado State University, pp.29.1-29.38.

3. Brown, C. B., 1943: Discussion of Sedimentation in Reservoirs, by J. Witzig. Proceedings of the American Society of Civil Engineers 69,1493-1500.

4. Brune, G. M., 1953. Trap Efficiency of Reservoirs. American Geophysical Union, 34(3), pp. 407-419.

5. Churchill, M. A., 1948: Discussion of Analyses and Use of Reservoir Sedimentation databy L.C. Gottschalk. In Proceedings of the federal interagency sedimentation conference, Denver, Colorado, Washington, DC: US Geological Survey, pp 139-40.

6. Gert Verstraeten and Jean Poesen, 2000. Estimating Trap Efficiency of Small Reservoirs and Ponds: Methods and Implications for the Assessment of Sediment Yield,. Progress in Physical Geography, 2 (24), pp. 219-251.

7. Gregory, L., Morris and Jiahua Fan, 1998. Reservoir Sedimentation Handbook. New York: McGraw-Hill Book Co.

8. Harbor, J., Bhaduri, B., Angelakis, L. and Snyder, J. 1997: Sediment basins, using modified stormwater management basins and sediment basins to reduce water pollution from construction sites in Ohio. Kent, $\mathrm{OH}$ Department of Geology, Kent State University.

9. Siyam, A. M., 2000: Reservoir Sedimentation Control. Ph.D. Thesis, University of Bristol, England.

10. Stephen, E. Lewis, Zoe, T. Bainbridge, Petra, M. Kuhnert, Bradford, S. Sherman, Brent Henderson, Cameron Dougall, Michelle Cooper, and Jon E. Brodie. 2013 : Calculating Sediment Trapping Efficiencies for Reservoirs in Tropical Settings: A Case Study from the Burdekin Falls Dam, NE Australia,Water Resources Research, Vol. 49, pp1017-1029.

11. Toniolo, H., and Schultz, J., 2005. Experiments on Sediment Trap Efficiency in Reservoirs. Lakes $\mathcal{E}$ Reservoirs: Research and Management 2005, vol 10, pp13-24

12. Trimble, S. W., and Carey, W. P., 1990: A Comparison of the Brune and Churchill Methods for Computing Sediment Yields Applied to a Reservoir System. USGS Water Supply Paper 2340, 195-202. 